\title{
Anaphylactic Reactions to Bee-Sting Challenges in Allergic Children Are Not Modified by Endogeneous Catecholamines
}

\author{
PIA HAUK, JOHANNES OTTO, K. OTFRIED SCHWAB, KLAUS KAUFMEHL, RADVAN \\ URBANEK, HANS GEORG EICHLER, AND JOHANNES FORSTER \\ University Children's Hospitals, Freiburg, Germany [P.H., K.K., J.F.] and Vienna, Austria [R.U.]; \\ Fachklinik fuer Kinder und Jugendliche, Wangen im Allgaeu, Germany [J.O.]; Department of \\ Pharmacology, University of Wuerzburg, Germany [K.O.S.]; Department of Clinical Pharmacology, \\ University of Vienna, Vienna, Austria [H.G.E.]
}

\begin{abstract}
ABST
To investigate the role of basal catecholamine levels and the
response of the adrenergic system to expected bee stings, plasma
catecholamines were measured before and 1 and 2 min after
bee-sting challenges. Twenty-one children (aged $4-15$ y) with
bee-sting allergies were selected for sequential challenges to
establish the need for venom immunotherapy. The time interval
between the challenges varied from 2 to 6 wh. Epinephrine,
norepinephrine, and dopamine plasma levels were measured
using a simultaneous single-isotope radioenzymatic assay. On
the first challenge, $33 \%$ of the children experienced a normal
local reaction, $29 \%$ a large local reaction, and 38\% a systemic
reaction. On the second challenge in 18 out of 21 subjects, $67 \%$
experienced a normal normal local reaction, $22 \%$ a large local
reaction, and $11 \%$ a systemic reaction. Epinephrine and norepi-
nephrine plasma levels increased significantly on the first and
second challenges. Dopamine plasma levels showed a significant
increase on the first challenge only. Plasma catecholamine levels
\end{abstract}
Between 0.4 and $4 \%$ of the population experience anaphylactic reactions to insect stings (1-3). Sensitization to insect venom can be verified using various diagnostic methods. However, numerous studies have been unsuccessful in showing a correlation between the findings of standard diagnostic methods - mainly skin-prick tests and the measurement of specific IgE and IgG antibodiesand the reaction to subsequent insect stings (4-7). The benign natural history of allergy to Hymenoptera stings particularly observed in childhood $(8-11)$ requires more precise scrutiny before an individual child can be assigned to receive venom immunotherapy. Single $(4,12)$ or sequential sting challenges $(13,14)$ can be offered to estimate the patient's individual risk and thus to establish the necessity for venom immunotherapy.

Various studies demonstrated a release of E, NE, and angiotensin II within several minutes after the onset of anaphylactic shock to

Received February 6, 1995; accepted June 30, 1995.

Correspondence: Pia Hauk, M.D., National Jewish Center for Immunology and Respiratory Medicine, Dept. of Pediatrics, Room K 1026A, 1400 Jackson St., Denver, CO 80206. after the second challenge revealed a significant positive correlation between epinephrine increases measured 1 and 2 min after the challenge and the concomitant sting reaction. Basal epinephrine, norepinephrine, and dopamine plasma levels did not differ significantly between patients who experienced different types of sting reactions. Based on our data, we conclude that clinical reactions to in-hospital insect-sting challenges are not affected by early increases in plasma catecholamine levels produced by the expected stress situation. (Pediatr Res 38: 998-1002, 1995)

\section{Abbreviations}

LR, local reaction

SR, systemic reaction

E, epinephrine

NE, norepinephrine

D, dopamine maintain cardiovascular functions (15-18). Van der Linden et al. (12) made the same observation when investigating insect sting-allergic patients using stings from the insects in question.

We asked the question whether anxiety about the subsequent diagnostic challenge could induce an early catecholamine release. It could be possible that increases in $\mathrm{E}$ and $\mathrm{NE}$ plasma concentrations occurring shortly before the challenge may affect the immediate reaction of the patient, thus diminishing the predictive value of the challenge. Therefore, the aim of our study was to measure catecholamine plasma concentrations shortly before and after challenge stings to investigate the influence of the sympatho-adrenergic system on patients' responses and to evaluate the validity of diagnostic stings.

\section{METHODS}

Patients. Twenty-one children, 16 boys and 5 girls aged 4-15 y (median 9 y), with "bee-sting allergies," together with 
their parents, gave informed consent to their participation in the study. They initially opted for sequential bee-sting challenges to determine the necessity for venom immunotherapy (14). The procedure was approved by the Medical Ethics Committee of the University, and informed consent was obtained from the parents, particularly with respect to the possible booster effect.

Classification of hypersensitivity reactions. Large LR $=$ an LR with redness and swelling $>10 \mathrm{~cm}$ in diameter and lasting longer than $24 \mathrm{~h}$; moderate $\mathrm{SR}=$ skin symptoms (general urticaria, redness, itching), angioedema, and gastrointestinal symptoms; severe SR = respiratory symptoms (wheezing, shortness of breath) and mainly cardiovascular symptoms (hypotension, collapse, cardiac arrhythmias).

Venom skin testing. Skin-prick testing was performed with purified bee (Apis mellifera) and wasp (Vespulae germanica et vulgaris) venom (ALK-prick SQ; ALK/Scherax, Hamburg, Germany) on the flexor surface of the forearm at concentrations of $0.1,1,10$, and $100 \mu \mathrm{g} / \mathrm{mL}$. Additional testing with wasp venom was performed to verify the relevance of the sensitization to bee venom. Negative (diluent) and positive (histamine, $10 \mathrm{mg} / \mathrm{mL}$ ) controls were also applied. The diameter of the resulting wheal was measured 20 min after the test was performed. At a venom concentration of $100 \mu \mathrm{g} / \mathrm{mL}$, a wheal diameter of $<3 \mathrm{~mm}$ was considered positive and $\geq 3 \mathrm{~mm}$ strongly positive.

Antibody measurements. IgE antibodies to bee venom were measured using the RAST technique (Pharmacia, Sweden) and were expressed as RAST classes 1-4. IgG antibodies were measured using the ELISA technique (19). Results were expressed as being low $(<400)$ or high ( $\geq 400$ arbitrary units).

Measurement of plasma catecholamines. E, NE, and D were measured using a modified single-isotope radioenzymatic assay according to the principles of Peuler and Johnson (20). The most important modification was deproteinization of plasma with $9 \mathrm{~N}$ perchloric acid, which allowed the determination of catecholamines in plasma using a standard curve (21-23). The lowest level of detection was 3-13 pmol/L. The interassay variance was below $12 \%$, the intraassay variance below $6 \%$ for all respective catecholamines.

Blood samples were taken from 13 out of 21 subjects during both challenge procedures, from 5 out of 21 during the first challenge only, and from 3 out of 21 during the second challenge only. Plasma samples were kept on ice after immediate separation at $4^{\circ} \mathrm{C}$. They were then stored at $-20^{\circ} \mathrm{C}$ before being transported (on dry ice) to the laboratory where the assay was performed.

Study protocol. The initial evaluation consisted of medical and allergic history, with emphasis on prior insect-sting reactions, a physical examination, venom skin testing, and venomspecific $\operatorname{IgE}$ and $\operatorname{IgG}$ antibody measurements. An intravenous cannula was inserted into a forearm vein. The subjects rested in a supine position for $15 \mathrm{~min}$, after which a blood sample was taken to measure basal catecholamine concentrations. The subjects then assumed a sitting position and their blood pressure, heart rate, and peak expiratory flow (Miniwright peak flow meter) were recorded.
Immediately after this, the subjects remained seated while the sting challenge was carried out. This was done by placing a living bee (A. mellifera) on the flexor side of the forearm contralateral to the intravenous cannula. Further blood samples for the measurement of catecholamines were taken 1 and 2 min after the challenge. Blood pressure and heart rate were recorded routinely at 5-15-min intervals when patients' conditions were stable and at shorter intervals when they were unstable. Additional measurements were taken if bronchial obstruction was suspected. A decrease in the peak expiratory flow of $\geq 15 \%$ was considered to be significant.

A second challenge was omitted, and venom immunotherapy started if the patient experienced a severe SR on the first challenge, and his or her parents agreed with the recommendation for treatment.

Statistical analysis. A statistical analysis was carried out using $t$ test for matched pairs (SPSS-PC-program) to evaluate the extent of changes in catecholamine plasma concentrations. An analysis of variance was performed to detect differences between patients experiencing different types of clinical reactions to the stings with respect to basal catecholamine levels and changes of plasma catecholamine levels after the challenges.

\section{RESULTS}

Twenty-four (23.8) percent of the children had reported large LRs, $23.8 \%$ moderate systemic, and $52.4 \%$ severe SR to the index bee sting. Twenty-one subjects were sensitized to bee venom which was verified by a positive skin-prick test and/or the presence of specific IgE antibodies to bee venom (data not shown in detail). Specific IgG levels to bee venom were high $\geq 400$ arbitrary units in $28.6 \%$ (6 out of 21 ) and low $(<400$ arbitrary units) in $66 \%$ (14 out of 21$)$. In one subject these levels were not measured.

Clinical reactions to bee-sting challenges. The severity and frequency of clinical reactions to the first and second challenges are shown in Fig. 1. The sequential challenge procedure was restricted to the first sting in three patients because they had developed a severe SR with bronchial obstruction and venom immunotherapy was therefore recommended. These 3 patients were 3 out of 11 subjects who have had severe index sting reactions. Blood pressure and heart rate remained stable in all individuals before and after the challenges (data not shown). Peak expiratory flow was significantly decreased in 2 out of 21 patients who had experienced severe SRs (data not shown in detail).

Plasma catecholamines. The mean concentrations of plasma catecholamines before and after sting challenges are shown in Table 1. For the first time challenge, blood samples were not available from three patients. For the second time challenge, blood samples were not available from five patients: in three patients because they were not restung.

There was no significant difference between basal values measured before the first and before the second challenge. In two patients, high basal NE levels (3103 and $3310 \mathrm{pmol} / \mathrm{L}$ ) were observed before the first challenge. In one of those patients, an elevated basal NE level (3007 pmol/L) was also 


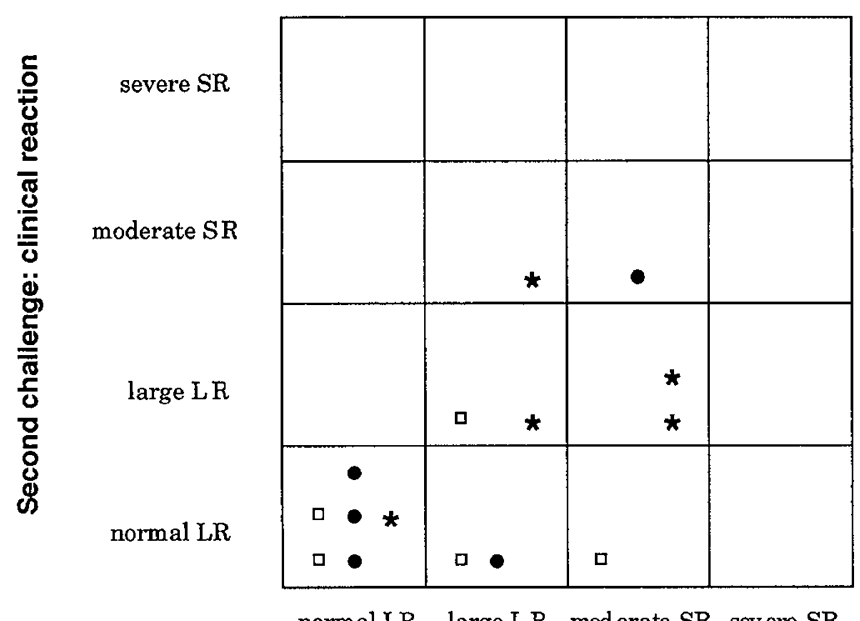

normal LR large LR moderate SR severe SR

First challenge: clinical reaction

Figure 1. Clinical reactions to bee-sting challenges. The clinical reactions of 18 out of 21 patients to the first and second sting challenge are shown. $\square=$ large LR as index sting; - moderate SR as index sting; * = severe SR as index sting.

Table 1. Mean concentration of plasma catecholamines and their range before and after challenges

\begin{tabular}{|c|c|c|c|}
\hline & $\begin{array}{c}\text { Before: } \\
\text { mean (range) } \\
(\text { pmol/L) }\end{array}$ & $\begin{array}{l}\text { One minute after: } \\
\text { mean (range) } \\
(\mathrm{pmol} / \mathrm{L})\end{array}$ & $\begin{array}{c}\text { Two minutes after: } \\
\text { mean (range) } \\
(\text { pmol/L) }\end{array}$ \\
\hline \multicolumn{4}{|c|}{ First challenge§ } \\
\hline $\mathrm{E}$ & $350(207-637)$ & $545(267-1655)^{* *}$ & $527(227-1096)^{* *}$ \\
\hline NE & $1802(1077-3310)$ & $2431(1787-4330)^{* *}$ & $2298(1200-4165)^{* *}$ \\
\hline $\mathrm{D}$ & $302(170-653)$ & $368(220-918)^{* *}$ & $355(153-808)^{*}$ \\
\hline \multicolumn{4}{|c|}{ Second challengeף } \\
\hline $\mathrm{E}$ & $387(179-775)$ & $660(222-2119)^{*}$ & $577(245-1054)^{* * *}$ \\
\hline $\mathrm{NE}$ & $1696(1020-3007)$ & $2306(1481-4398)^{* *}$ & $2159(1282-3497)^{* *} \dagger$ \\
\hline $\mathrm{D}$ & $340(162-1109)$ & $381(180-964)$ & $381(188-838)$ \\
\hline
\end{tabular}

Significant catecholamine increase compared to basal value $(* *=p<0.01$, $*=p<0.05$ ).

$\uparrow$ Significant decrease between 1 and 2 min after challenge $(p<0.05)$.

$\S$ Data from 18 patients.

II Data from 16 patients.

observed before the second challenge. Plasma from the other patient was not examined during the second challenge procedure. The reaction to the sting challenge did not correlate with any initial catecholamine level.

Plasma E and NE levels increased significantly after both challenges. Elevated plasma NE levels showed a significant decrease from 1 to $2 \mathrm{~min}$ after the second challenge. Mean plasma D levels showed a significant increase on the first challenge. D levels were not significantly affected by the second challenge (Table 1).

Percentage increases of plasma E, NE, and D in individual patients resulting from the first and second challenges are shown in Figs. 2 and 3, respectively. Basal values were calculated as $100 \%$.

Changes in plasma catecholamine levels after the first challenge did not correlate with the concomitant sting reaction. Plasma catecholamine levels after the second challenge revealed a significant correlation $(p<0.05)$ between $\mathrm{E}$ increases measured one and $2 \mathrm{~min}$ after the challenge and the concom-

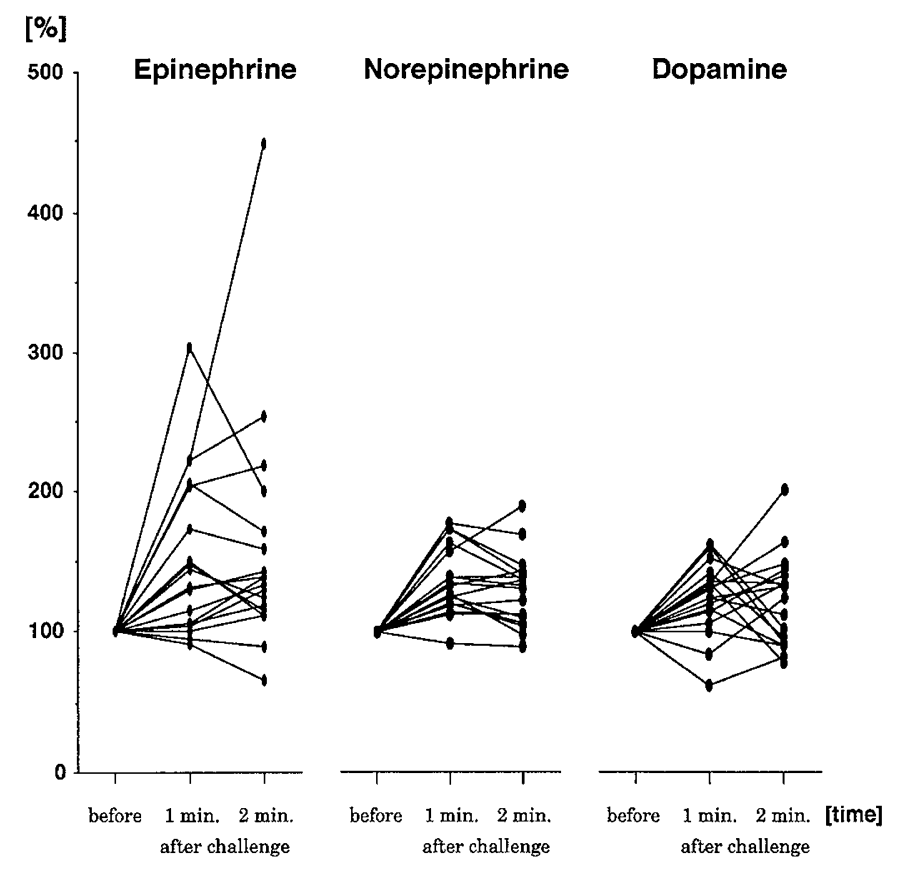

Figure 2. Catecholamine increase in individual patients on first challenge. Percentage increase 1 and 2 min after challenge compared with basal value $(=100 \%)$. Data from 18 patients are shown.

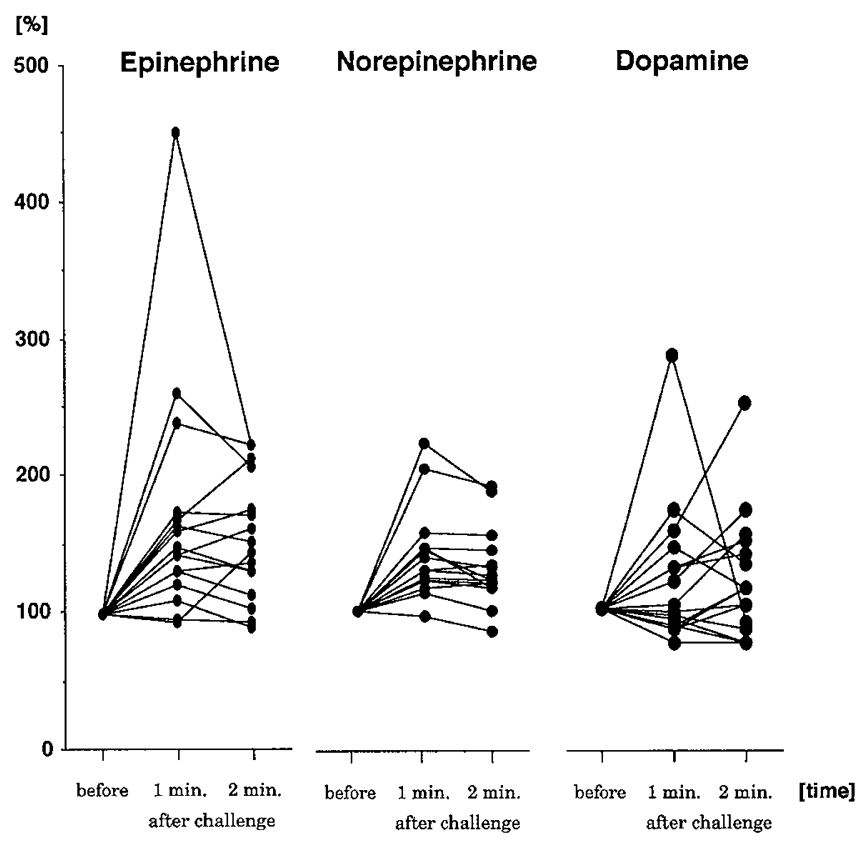

Figure 3. Catecholamine increase in individual patients on second challenge. Percentage increase 1 and 2 min after challenge compared with basal value $(=100 \%)$. Data from 16 patients are shown.

itant sting reaction. Patients who experienced a more severe allergic reaction demonstrated higher increases in plasma $\mathrm{E}$ (Table 2).

\section{DISCUSSION}

Our hypothesis was that an elevated plasma catecholamine level caused by patients' anticipating an in-hospital insect sting immediately before it is given could influence the subsequent 
Table 2. The type of clinical reaction to second sting challenge correlated with the concomitant $E$ increase in challenged patients

\begin{tabular}{|c|c|c|c|}
\hline \multirow[b]{2}{*}{$\begin{array}{l}\text { Clinical reaction to } \\
\text { second challenge }\end{array}$} & \multirow[b]{2}{*}{$(n)$} & \multicolumn{2}{|c|}{ E increase } \\
\hline & & $\begin{array}{l}\text { One minute after } \\
\text { second challenge: } \\
\text { (mean (range) }) \\
(\mathrm{pmol} / \mathrm{L})\end{array}$ & $\begin{array}{l}\text { Two minutes after } \\
\text { second challenge: } \\
\text { (mean (range) } \\
(\mathrm{pmol} / \mathrm{L})\end{array}$ \\
\hline Normal LR & (11) & $124(-19-326)^{*}$ & $128(-40-401)^{*}$ \\
\hline Large LR & (3) & $376(307-481)^{*}$ & $257(199-324)^{*}$ \\
\hline Moderate SR & (2) & $937(222-1651)^{*}$ & $432(277-586)^{*}$ \\
\hline Total & 16 & & \\
\hline
\end{tabular}

$* p<0.05$.

sting reaction. By measuring plasma $\mathrm{E}, \mathrm{NE}$, and D levels shortly before and one and 2 min after a sting, it should be possible to detect any such early elevation, as plasma catecholamines have a brief half-life of about 2 min (24).

Blood samples for the measurement of basal values were taken $15 \mathrm{~min}$ after inserting the intravenous cannula, because it is well known that differences in test results can occur depending on when blood samples are taken after insertion of a cannula (25). Basal E levels measured before the first and second challenges were not elevated. Our data were comparable to the age-dependent $E$ values in resting, nonsedated children published by Planz et al. (26) obtained using the radioenzymatic method for the determination of $E, N E$, and $D$. They were also comparable to E levels in blood samples taken from healthy infants and children $30 \mathrm{~min}$ after venipuncture which were measured by Eichler et al. (27) using the HPLC method. Basal NE levels measured before both challenges also varied within the normal range for healthy children as previously described $(26,27)$. Slightly elevated basal NE levels were found before the first challenge in only two patients and in one of those two before the second challenge also. These slightly elevated NE levels may also be regarded as normal, because there is a lack of age-dependent reference values for plasma catecholamines in healthy children. Basal D levels were within the normal range for healty adults determined by HPLC (27) in all patients except one, who had shown a slight elevation before the second challenge. Eichler et al. (27) suggested that, for $\mathrm{D}$, adult reference levels are applicable to any age group, as no child tested except one had levels exceeding $653 \mathrm{pmol} / \mathrm{L}$. No reference data on healthy children measured using the radioenzymatic method are available. Thus from the measured values, catecholamines could not be expected to affect the challenge reaction. Indeed, we found no correlation between those levels and the degree of the allergic reaction.

Plasma $\mathrm{E}$ and NE levels increased significantly after both challenges, but the increased levels either varied within the ranges normal for healthy infants $(26,27)$ or were slightly elevated. Slight but significant increases of plasma D levels were measured after the first challenge sting only.

Previous investigations $(28,29)$ have demonstrated that different stimuli caused different neuronal and adrenomedullary catecholamine release in man. Exercise seems to induce a response from the sympathetic nervous system which causes an increase in plasma NE, whereas psychological stress primarily induces an adrenal response resulting in elevated plasma $\mathrm{E}$ levels. The knowledge that they would receive an in-hospital bee sting, which is a type of mental stress, did not affect catecholamine plasma levels in our patients, and in particular did not increase E. Based on our data, sting challenges cannot only be interpreted as purely mental stress, as not only E, but also NE increases significantly. Sting challenges therefore appear to represent a combination of mental and physical stress which causes increases in both $\mathrm{E}$ and NE. In our study, changes in plasma D levels occurred on a smaller scale, which is explained by the fact that $D$ is a precursor of $E$ and $N E$ which is generally liberated to a certain extent due to stress.

The evaluation of the severity of sting reactions with respect to changes in plasma catecholamine levels after challenge stings only revealed a significant correlation between clinical reactions and $\mathrm{E}$ increase 1 and $2 \mathrm{~min}$ after the second challenge. A study by Clutter et al. (30) demonstrated plasma E thresholds of 273-546 pmol/L for increments in heart rate, of 409-682 pmol/L for increments in systolic blood pressure and of 819-1092 $\mathrm{pmol} / \mathrm{L}$ for decreases in diastolic blood pressure. Although some of our patients reached E levels within those ranges shortly after the challenge, no significant changes in heart rate and blood pressure were observed at any time. In contrast, van der Linden et al. (12) reported a correlation between increases in $\mathrm{E}, \mathrm{NE}$, and angiotensin II and a drop in blood pressure within $5 \mathrm{~min}$ after the onset of anaphylactic symptoms due to an insect-sting challenge. However, these data cannot be compared with our results, as the van der Linden study investigated the correlation between catecholamine release and clinical reaction after the onset of anaphylactic symptoms.

In our study, bee venom-allergic children investigated using sequential sting challenges were not endagered by lifethreatening anaphylactic reactions. Children who had lower catecholamine levels before the challenges did not develop more severe reactions to the subsequent sting than those with higher basal catecholamine levels. Based on our data, we conclude that the outcome of in-hospital insect-sting challenges is not affected by early increases in plasma catecholamines resulting from the expected stress situation. It has thus been possible to better verify the validity of diagnostic sting challenges.

\section{REFERENCES}

1. Golden BK, Marsh DG, Kagey-Sobotka A, Freidhoff L, Szklo M, Valentine MD, Lichtenstein LM 1989 Epidemiology of insect venom sensitivity. JAMA 262:240244

2. Kampelmacher MJ, van der Zwan JC 1987 Provocation test with a living insect as a diagnostic tool in systemic reactions to bee or wasp venom: a prospective study with emphasis on clinical aspects. Clin Allergy 17:317-327

3. Settipane GA, Chafee FH 1979 Natural history of allergy to Hymenoptera. Clin Allergy 9:385-390

4. Blaauw PJ, Smithuis LOMJ 1985 The evaluation of the common diagnostic methods of hypersensitivity for bee and yellow jacket venom by means of an in-hospital insect sting. J Allergy Clin Immunol 75:556-562

5. Heinig JH, Engel T, Weeke ER 1988 Allergy to venom from bee or wasp: the relation between clinical and immunological reactions to insect stings. Clin Allergy 18:71-78

6. Parker JL, Santrach PJ, Dahlberg MJE, Yuninger JW 1982 Evaluation of Hymenoptera-sting sensitivity with deliberate sting challenges: inadequacy of present diagnostic methods. J Allergy Clin Immunol 69:200-207

7. Reisman RE, Lazell M, Arbesman CE 1981 Insect venom allergy: a prospective case showing the lack of correlation between immunologic reactivity and clinical sensitivity. J Allergy Clin Immunol 68:406-408

8. Graft DF, Schuberth KC, Kagey-Sobotka A, Kwiterovich KA, Niv Y, Lichtenstein LM, Valentine MD 1984 A prospective study of the natural history of large local reactions after Hymenoptera stings in children. J Pediatr 104:664-668 
9. Schuberth KC, Lichtenstein LM, Kagey-Sobotka A, Szklo M, Kwiterovich KA Valentine MD 1983 Epidemiologic study of insect allergy in children. II. Effect of accidental stings in allergic children. J Pediatr 102:361-365

10. Valentine MD, Golden DBK 1986 Diagnosis of insect allergy. In: Levine MI, Lockey RF (eds) Monograph on Insect Allergy. Lambert Assoc., Pittsburgh, pp 39-43

11. Valentine MD, Schuberth KC, Kagey-Sobotka A, Graft FG, Kwiterovich KA, Szklo M, Lichtenstein LM 1990 The value of immunotherapy with venom in children with allergy to insect stings. N Engl J Med 323:1601-1603

12. Van der Linden PWG, Struyvenberg A, Kraaijenhagen RJ, Hack CE, van der Zwan JK 1993 Anaphylactic shock after insect-sting challenge in 138 persons with a previous insect-sting reaction. Ann Intern Med 118:161-168

13. Franken HH, Dubois AEJ, Minkema HJ, van der Heide S, de Monchy JGR 1994 Lack of reproducibility of a single negative sting challenge response in the assessment of anaphylactic risk in patients with suspected yellow jacket hypersensitivity. J Allergy Clin Immunol 93:431-436

14. Hauk P, Friedl K, Kaufmehl K, Urbanek R, Forster J 1995 Subsequent insect stings in children with Hymenoptera hypersensitivity. J Pediatr 126:185-190

15. Zavecz JH, Levi R 1977 Separation of primary and secondary cardiovascular events in systemic anaphylaxis. Circ Res 40:15-19

16. Pavec K, Wegmann A, Nordström L, Schwander D 1982 Cardiovascular and respiratory mechanisms in anaphylactic and anaphylactoid shock reactions. Klin Wochenschr 60:941-947

17. Levi R 1988 Cardiac anaphylaxis: models, mediators, mechanisms and clinical considerations. In: Marone G, Lichtenstein LM, Condorelli M, Fauci AS (eds) Human Inflammatory Disease. Clinical Immunology, Vol. 1. Decker BC Inc., Philadelphia, pp $93-105$

18. Piper PJ, Collier HOJ 1967 Release of catecholamines in the guinea-pig by substances involved in anaphylaxis. Nature 213:838-840

19. Forster J, Urbanek R 1979 Enzyme-linked immunosorbent assay of allergen-specific IgG antibodies in bee sting allergic patients hyposensitized with pure bee venom. Klin Wochenschr 57:431-432
20. Peuler JD, Johnson GA 1977 Simultaneous single isotope radioenzymatic assay of plasma norepinephrine, epinephrine and dopamine. Life Sci 21:625-636

21. Schwab KO, Kruse K, Breitung B, Paulick R 1989 Free and sulfoconjugated catecholamines in preterm and term newborns after delivery. Monatsschr Kinderheilkd 137:28-32

22. Schwab KO, Heubel G, Bartels H 1992 Free epinephrine, norepinephrine and dopamine in saliva and plasma of healthy adults. Eur J Clin Chem Clin Biochem 30:541-544

23. Schwab KO, von Stockhausen HB 1994 Plasma catecholamines after endotracheal administration of adrenaline during postnatal resuscitation. Arch Dis Child 70:F213F217

24. FitzGerald GA, Hossmann V, Hamilton CA, Reid JL, Davies DS, Dollery CT 1979 Interindividual variation in kinetics of infused epinephrine. Clin Pharmacol Ther 26:669-675

25. Pluto R, Buerger $\mathrm{P}$, Weicker $\mathrm{H} 1986$ The physiological variability of catecholamine levels in plasma. Klin Wochenschr 64:625-632

26. Planz G, Bieber J, Mencke HJ, v. Bernuth G 1983 Plasma catecholamines in children. Experientia 39:871

27. Eichler I, Eichler H-G, Rotter M, Kyrle PA, Gasic S, Korn A 1989 Plasma concentrations of free and sulfoconjugated dopamine, epinephrine, and norepinephrine in healthy infants and children. Klin Wochenschr 67:672-675

28. Robertson D, Johnson GA, Robertson RM, Nies AS, Shand DG, Oates JA 1979 Comparative assessment of stimuli that release neuronal and adrenomedullary catecholamines in man. Circulation 59:637-643

29. Dimsdale JE, Moss J 1980 Plasma catecholamines in stress and exercise. JAMA 243:340-342

30. Clutter WE, Bier DM, Suresh DS, Cryer PE 1980 Epinephrine plasma metabolic clearance rates and physiologic thresholds for metabolic and hemodynamic actions in man. J Clin Invest 66:94-101 